\title{
Ocorrência de parasitóides e patógenos em adultos de Chrysomelidae (Coleoptera) em estufa plástica, em Santa Maria, RS
}

\author{
Occurrence of parasitoids and pathogens on adults of Chrysomelidae (Coleoptera) in greenhouse, in \\ Santa Maria, RS
}

\author{
Sônia Thereza Bastos Dequech ${ }^{1}$ Carla Daniele Sausen² Juliano Dalcin Martins ${ }^{2}$ \\ Cíntia Grendene Lima² Rafael Egewarth ${ }^{2}$ Roberta Cristina Zumba ${ }^{2}$ \\ Jerson Vanderlei Carús Guedes ${ }^{3}$
}

\begin{abstract}
- NOTA -
RESUMO

Estufas plásticas representam uma alternativa para o cultivo de olerícolas sob condições climáticas desfavoráveis. O pepino (Cucumis sativus L.) está entre as hortícolas mais cultivadas nesses ambientes protegidos. Dentre os seus principais insetos-praga, destacam-se os crisomelídeos. O objetivo deste trabalho foi identificar os inimigos naturais de adultos desses insetos em cultivo de pepino em ambiente protegido. $O$ pepino foi plantado na primeira semana de março de 2003, em estufa plástica situada em área experimental da Universidade Federal de Santa Maria, em Santa Maria, Rio Grande do Sul, Brasil. Os crisomelídeos foram coletados a cada dois ou três dias por semana durante todo o período de cultivo, totalizando 28 datas de coleta. Nas primeiras 22 amostragens, foram coletadas 50 vaquinhas/data e, nas demais, 30/data. As espécies de crisomelídeos identificadas foram: Diabrotica speciosa (Germar), Cerotoma arcuata Olivier e Acalymma bivittula (Kirsch). Celatoria bosqi Blanchard (Diptera: Tachinidae) foi a única espécie de parasitóide identificada, parasitando $\boldsymbol{D}$.

horticultural crops in these protected environments. Among its main insect pests are the chrysomelid beetles. The objective of the present research was to determine the natural enemies of chrysomelids in cucumber grown in protected environment. The cucumber was seeded on the first week of March, 2003, in plastic greenhouse located in the experimental area of the Federal University of Santa Maria, in Santa Maria, Rio Grande do Sul, Brazil. Chrysomelids were collected every two or three days/week during the whole cultivation period, totalizing 28 collection dates. In the first 22 samplings, 50 chrysomelids/ date were collected and, in the others, 30/date. The species of chrysomelids identified were: Diabrotica speciosa (Germar), Cerotoma arcuata Olivier and Acalymma bivittula (Kirsch). Celatoria bosqi Blanchard (Diptera: Tachinidae) was the only parasitoid specie identified parasitizing most frequently $\boldsymbol{D}$. speciosa. Beauveria bassiana (Bals.) Vuill. infected adults of D. speciosa and A. bivittula, and the first specie was more susceptible. This is the first record of the occurrence of $\boldsymbol{C}$. bosqi and B. bassiana on chrysomelids in crops cultivated in protected environment.
\end{abstract} speciosa com maior freqüência. Beauveria bassiana (Bals.) Vuill. infectou adultos de D. speciosa e de A. bivittula, sendo que a primeira espécie se mostrou mais suscetível. Este é o primeiro registro da ocorrência de $\boldsymbol{C}$. bosqi e B. bassiana em crisomelídeos em ambiente protegido.

Palavras-chave: Celatoria bosqi, Beauveria bassiana, Cucumis sativus.

\section{ABSTRACT}

Greenhouses represent an alternative for horticultural crops when the climatic conditions are unfavorable. Cucumber (Cucumis sativus L.) is one of the most cultivated
Key words: Celatoria bosqi, Beauveria bassiana, Cucumis sativus.

A utilização de estufas plásticas para o cultivo comercial de espécies olerícolas representa a possibilidade de colheitas em períodos de entressafra, por proteger as culturas dos fatores climáticos que são adversos para a sua exploração em ambiente natural, isto é, a campo. Esta é uma prática que vem sendo bastante utilizada na região central do Estado do Rio

${ }^{1}$ Departamento de Defesa Fitossanitária, Universidade Federal de Santa Maria (UFSM), 97105-900, Santa Maria, RS, Brasil. Email: soniabd@terra.com.br. Autor para correspondência.

${ }^{2}$ Curso de Agronomia, UFSM, Santa Maria, RS, Brasil.

${ }^{3}$ Departamento de Defesa Fitossanitária, UFSM, Santa Maria, RS, Brasil. 
Grande do Sul, sendo que, na região de abrangência da EMATER Regional de Santa Maria, a área protegida por túneis altos e estufas foi de 21,5ha, com 947 unidades utilizadas por 483 produtores, no ano de 2000 . São cultivados, basicamente, tomate tipo salada, alface, pepino para conserva, feijão de vagem e melão (POERSCHKE, 2003 - Informe verbal).

Os coleópteros da família Chrysomelidae, conhecidos popularmente como vaquinhas, causam danos significativos em folhas e flores e são encontrados com freqüência em cultivos de cucurbitáceas, solanáceas e fabáceas, incluindo feijoeiro (SALAS et al., 1999; NAVA, 2000; GALLO et al., 2002). Todavia, poucas são as informações sobre os inimigos naturais de crisomelídeos, especialmente em condições de ambiente protegido. Citações sobre parasitóides desses insetos, particularmente de Diabrotica speciosa e/ou Cerotoma arcuata, incluem SILVAet al. (1968), MAGALHÃES \& QUINTELA(1987), GASSEN et al. (1993), HEINECK-LEONEL \& SALLES (1997), CABRERA WALSH $(2004,2005)$ e MICHELI (2005). HEINECK-LEONEL \& SALLES (1997) e MICHELI (2005) referem-se, ainda, à ocorrência de fungos entomopatogênicos. O presente trabalho objetivou identificar as espécies de parasitóides e de patógenos de vaquinhas (Coleoptera: Chrysomelidae) em cultivo de pepino (Cucumis sativus L.), em condição de ambiente protegido.

As amostragens dos crisomelídeos foram realizadas em estufa plástica de $25 \mathrm{~m}$ x $8 \mathrm{~m}\left(200 \mathrm{~m}^{2}\right)$, com pé-direito de $2,50 \mathrm{~m}$, estrutura metálica e teto em arco, paredes laterais e frontais de polietileno de baixa densidade (PEBD) aditivado anti UV de 150 micra e cobertura de PVC transparente, antigotejo, pertencente ao Departamento de Defesa Fitossanitária (Centro de Ciências Rurais - CCR), situada no Campus da Universidade Federal de Santa Maria (UFSM), em Santa Maria, RS (latitude de $29^{\circ} 43^{\prime}$ S, longitude de $53^{\circ} 42^{\prime} \mathrm{W}$ e altitude de $\left.95 \mathrm{~m}\right)$.

O pepino foi plantado na primeira semana de março de 2003, com espaçamento de $1 \mathrm{~m}$ entre fileiras e de $0,30 \mathrm{~m}$ entre plantas, totalizando oito fileiras. As plantas foram conduzidas com fitas plásticas verticais e a cobertura do solo foi com plástico preto. A irrigação foi realizada por gotejamento, com as mangueiras colocadas sob o plástico preto.

As coletas de adultos de crisomelídeos foram realizadas manualmente a cada dois ou três dias por semana a partir do início de abril, totalizando 28 datas de coleta (de 04 de abril a 06 de junho). Nas primeiras 22 datas, foram coletadas, aleatoriamente, 50 vaquinhas/data e, nas demais, 30/data. Os insetos coletados foram transportados, em sacos plásticos, para o Laboratório de Entomologia do Departamento de Defesa Fitossanitária (CCR/UFSM), onde foram individualizados em potes plásticos (com capacidade de 100ml e tampas perfuradas para permitir ventilação) e alimentados com rodelas de cenoura crua, trocadas a cada três dias. As vaquinhas foram mantidas em câmara climatizada regulada para $25^{\circ} \mathrm{C}$, com umidade relativa mantida em aproximadamente $65 \%$ e com 12 horas de fotofase, sendo observadas diariamente quanto à emergência de parasitóides ou à presença de patógenos. Foi quantificada a contribuição da espécie de parasitóide ou patógeno como fator de mortalidade dos insetos hospedeiros através do cálculo da porcentagem de insetos parasitados ou doentes sobre o número total de indivíduos coletados de cada espécie de vaquinha.

Foram coletados 1.302 indivíduos, distribuídos em três espécies: $54,84 \%$ de Diabrotica speciosa (Germar), 22,89\% de Cerotoma arcuata Olivier e 22,27\% de Acalymma bivittula (Kirsch). Foi observada a emergência do parasitóide Celatoria bosqi Blanchard (Diptera: Tachinidae) em 4,76\%, 1,34\% e 0,69\%, respectivamente, dos adultos de D. speciosa, C. arcuata e A. bivittula. Foi encontrada, ainda, a presença do fungo entomopatogênico Beauveria bassiana (Bals.) Vuill. em 5,88\% dos indivíduos de $\boldsymbol{D}$. speciosa e em $0,34 \%$ de A. bivittula. Este é o primeiro registro tanto da ocorrência de $\boldsymbol{C}$. bosqi e B. bassiana em vaquinhas em ambiente protegido quanto da avaliação de inimigos naturais de $\boldsymbol{A}$. bivittula.

O período de amostragem (04 de abril a 06 de junho) restringiu-se, basicamente, aos meses de abril e maio de 2003, abrangendo o ciclo da cultura de pepino. Nesses meses, a maior ocorrência dos inimigos naturais avaliados foi durante o mês de maio, em $\boldsymbol{D}$. speciosa, com $5,78 \%$ dos indivíduos parasitados e $8,92 \%$ com fungo entomopatogênico, totalizando $14,70 \%$ dos indivíduos coletados naquele mês. As porcentagens de insetos, tanto parasitados quanto com patógenos, ficaram bem abaixo daquelas encontradas por HEINECK-LEONEL \& SALLES (1997), na região de Pelotas (RS), os quais chegaram a obter $30,2 \%$ dos indivíduos de $\boldsymbol{D}$. speciosa parasitados por $\boldsymbol{C}$. bosqi (em abril de 1995) e 13,9\% atacados por B. bassiana (março de 1995).

A maior porcentagem de $\boldsymbol{D}$. speciosa com parasitismo por $\boldsymbol{C}$. bosqi foi em 2/junho (15\%) (Figura 1), sendo inferior à encontrada por MICHELI (2005), que obteve parasitismo de $26 \%$ por Celatoria sp., em insetos coletados em lavouras de soja, em Londrina (PR). Já o maior ataque de B. bassiana foi em 26/maio $(22,86 \%)$ (Figura 1), ficando acima do valor encontrado por MICHELI (2005), que obteve $10 \%$ dos indivíduos com Beauveria sp. 


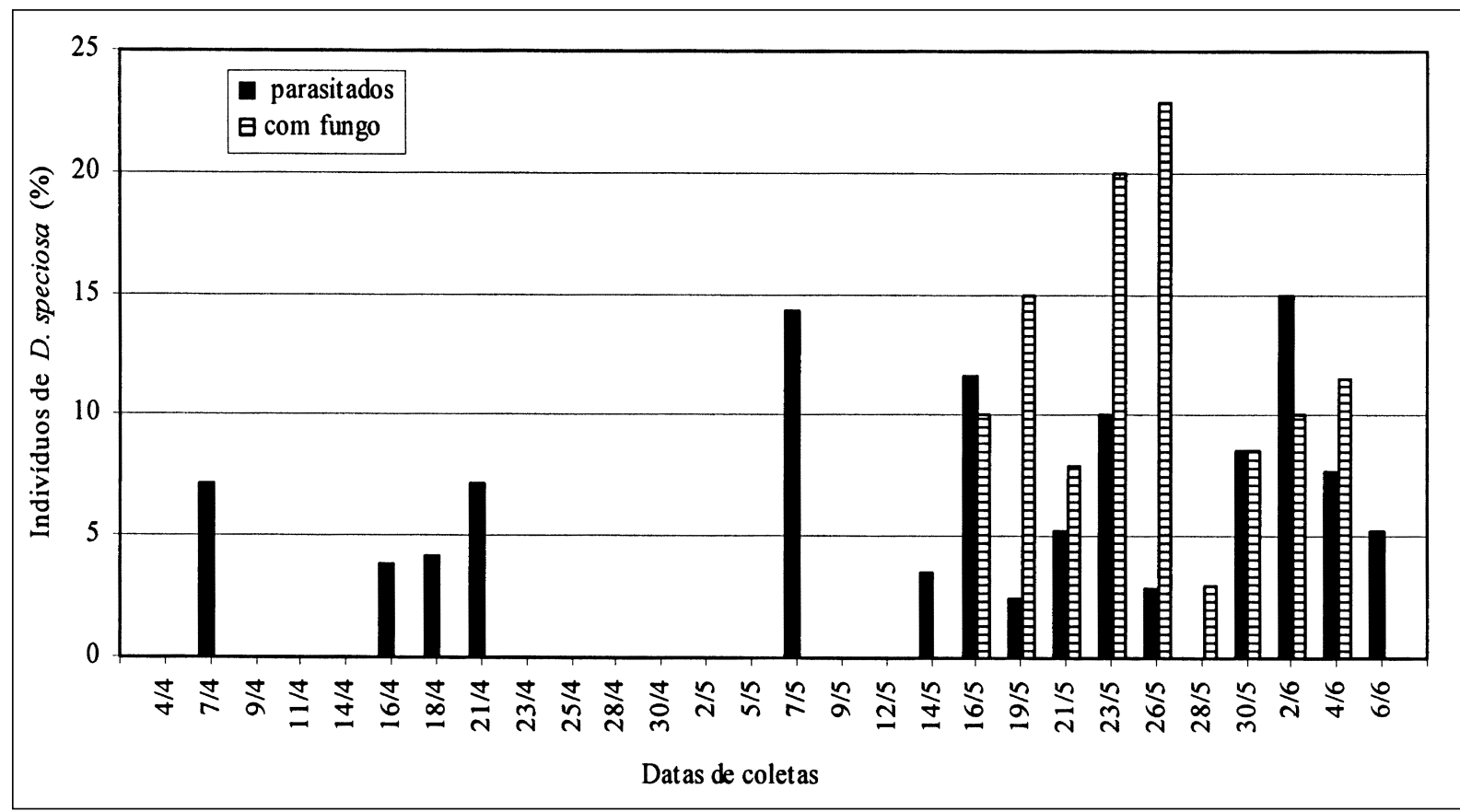

Figura 1 - Porcentagem de indivíduos de Diabrotica speciosa nas diferentes datas de coleta parasitados por Celatoria bosqi ou com a presença do fungo entomopatogênico Beauveria bassiana. Santa Maria, RS, 2003.

Parasitismo em $\boldsymbol{C}$. arcuata foi observado somente no mês de abril de 2003, correspondendo a $1,78 \%$ do total coletado no período. Este valor ficou muito abaixo daquele encontrado por MAGALHÃES \& QUINTELA (1987), que, em Goiás, encontraram 32,2\% de parasitismo por $\boldsymbol{C}$. bosqi sobre $\boldsymbol{C}$. arcuata, em março de 1985, em cultivo de caupi (Vigna unguiculata (L.) Walp.).

Destaca-se que a presença de indivíduos de $\boldsymbol{A}$. bivittula parasitados por $\boldsymbol{C}$. bosqi permite acrescentar este crisomelídeo às observações de CABRERA WALSH (2004) sobre os hospedeiros de $\boldsymbol{C}$. bosqi no Sul do Brasil, na Argentina, na Bolívia e no Uruguai. Assim, além de D. speciosa e C. arcuata, $\boldsymbol{A}$. bivittula também pode ser hospedeiro de $\boldsymbol{C}$. bosqi.

O baixo nível populacional tanto de parasitóides quanto de patógenos, encontrado nas vaquinhas avaliadas no presente trabalho, possivelmente deveu-se aos insetos terem sido coletados em estufas plásticas, cujo ambiente pode não ter sido favorável ao desenvolvimento dos inimigos naturais. Os valores obtidos indicam que $\boldsymbol{C}$. bosqi e B. bassiana não podem ser considerados eficientes como agentes de controle biológico natural de D. speciosa, C. arcuata e A. bivittula, porém apresentam potencial para serem avaliados visando a serem utilizados em programas de controle biológico desses crisomelídeos.
Portanto, pode-se concluir que, em pepino cultivado em ambiente protegido no município de Santa Maria, RS, $\boldsymbol{C}$. bosqi ocorre parasitando adultos de $\boldsymbol{D}$. speciosa, C. arcuata e A. bivittula, e B. bassiana, causando doença em $D$. speciosa e $A$. bivittula, porém em níveis muito baixos.

\section{AGRADECIMENTOS}

Os autores agradecem aos pesquisadores Dr. Fernando Zanotta da Cruz (Depto. de Fitossanidade, UFRGS, Porto Alegre, RS) e Dr. Ronaldo Toma (Museu de Entomologia, USP, São Paulo, SP) pela identificação de $\boldsymbol{A}$. bivittula e $\boldsymbol{C}$. bosqi, respectivamente, e ao Dr. Daniel Sosa-Gomez (Embrapa - CNPSo, Londrina, PR), pela identificação de B. bassiana.

\section{INFORMEVERBAL}

POERSCHKE, 2003. Eng. Agrônomo Paulo Renato Poerschke, Emater/RS Regional de Santa Maria, Av. Medianeira, 278, 97060-000, Santa Maria/RS, Brasil. E-mail: prenato@emater.tche.br

\section{REFERÊNCIAS}

CABRERA WALSH, G. Distribution, host specificity, and overwintering of Celatoria bosqi Blanchard (Diptera: Tachinidae), a South American parasitoid of Diabrotica spp. (Coleoptera: Chrysomelidae: Galerucinae). Biological Control, Orlando, v.29, n.3, p.427-434, 2004.

CABRERA WALSH, G. Corn rootworms. Capturado em 10 maio 2005. Online. Disponível na Internet http://www.usdasabcl.org/projects/CRW.htm\#top 
GALLO, D. et al. Entomologia agrícola. Piracicaba: FEALQ, 2002. 920p.

GASSEN, D.N. et al. Parasitismo em adultos de Diabrotica speciosa na região do Planalto do Rio Grande do Sul. In: REUNIÃO SUL-BRASILEIRA DE INSETOS DE SOLO, 4. 1993, Passo Fundo, RS. Anais e Ata... Passo Fundo: EMBRAPA/MAA/SEB, 1993. p.147-148. 176p.

HEINECK-LEONEL, M.A.; SALLES, L.A.B. Incidência de parasitóides e patógenos em adultos de Diabrotica speciosa (Germar, 1824) (Col., Chrysomelidae) na região de Pelotas, RS. Anais da Sociedade Entomológica do Brasil, Londrina, v.26, n.1, p.81-85, 1997.

MAGALHÃES, B.P.; QUINTELA, E.D. Níveis de parasitismo de Urosigalphus chalcodermi Wilkinson sobre Chalcodermus bimaculatus Fiedler e de Celatoria bosqi Blanchard sobre Cerotoma arcuata Olivier em caupi (Vigna unguiculata (L.) Walp.), em Goiás. Anais da Sociedade Entomológica do Brasil, Porto Alegre, v.16, n.1, p.235-238, 1987.

MICHELI, A. Variabilidade intraespecífica, inimigos naturais e avaliação da mistura de fungos entomopatogênicos e inseticidas para o controle de Diabrotica speciosa (Germar, 1824) (Coleoptera: Chrysomelidae). 2005. 115f. Dissertação (Mestrado em Ciências Biológicas) - Curso de Pós-graduação em Ciências Biológicas, Área de Concentração Entomologia, Universidade Federal do Paraná.

NAVA, D.E. Bioecologia de Cerotoma arcuatus Olivier, 1791 (Coleoptera, Chrysomelidae) e comprovação, em campo, do modelo de exigências térmicas obtido em laboratório. 2000. 72f. Dissertação (Mestrado em Ciências) - Curso de Pós-graduação em Ciências, Área de Concentração Entomologia, Escola Superior de Agricultura "Luiz Queiroz", Universidade de São Paulo.

SALAS, F.J.S. et al. Tentativas de transmissão de um isolado do vírus do mosaico severo do caupi (CpSMV-SP) por artrópodos, em laboratório. Scientia Agricola, Piracicaba, v.56, n.2, p.413-420, 1999.

SILVA, A.G. d'A. et al. Quarto catálogo dos insetos que vivem nas plantas do Brasil: seus parasitos e predadores. Rio de Janeiro: Ministério da Agricultura, 1968. 265p. 